\title{
Further $q$-supercongruences from a transformation of Rahman
}

\author{
Victor J. W. Guo \\ School of Mathematics and Statistics, Huaiyin Normal University, Huai'an 223300, Jiangsu, People's \\ Republic of China \\ jwguo@hytc.edu.cn
}

\begin{abstract}
Employing a quadratic transformation formula of Rahman and the method of 'creative microscoping' (introduced by the author and Zudilin in 2019), we provide some new $q$-supercongruences for truncated basic hypergeometric series. In particular, we confirm two recent conjectures of Liu and Wang. We also propose some related conjectures on supercongruences and $q$-supercongruences.
\end{abstract}

Keywords: cyclotomic polynomials; q-supercongruences; Rahman's transformation; creative microscoping

AMS Subject Classifications: 33D15; Secondary 11A07, 11B65

\section{Introduction}

In his first letter to Hardy on the 16th January 1913, Ramanujan mentioned the following formula:

$$
\sum_{k=0}^{\infty}(8 k+1) \frac{\left(\frac{1}{4}\right)_{k}^{4}}{k !^{4}}=\frac{2 \sqrt{2}}{\sqrt{\pi} \Gamma\left(\frac{3}{4}\right)^{2}}
$$

(see [1, p. 25, (2)]), where $(a)_{n}=a(a+1) \cdots(a+n-1)$ is the Pochhammer symbol and $\Gamma(x)$ denotes the Gamma function. Recently, Chen and Chu [2] gave a $q$-analogue of (1.1) as follows:

$$
\sum_{k=0}^{\infty}[8 k+1] \frac{\left(q ; q^{4}\right)_{k}^{4}}{\left(q^{4} ; q^{4}\right)_{k}^{4}} q^{2 k}=\frac{\left(q^{5}, q^{3}, q^{3}, q^{3} ; q^{4}\right)_{\infty}}{\left(q^{2}, q^{4}, q^{4}, q^{4} ; q^{4}\right)_{\infty}}
$$

They also obtained the following similar formula:

$$
\sum_{k=0}^{\infty}[6 k+1] \frac{\left(q ; q^{4}\right)_{k}\left(q ; q^{2}\right)_{k}^{3}}{\left(q^{2} ; q^{2}\right)_{k}\left(q^{4} ; q^{4}\right)_{k}^{3}} q^{k^{2}+k}=\frac{\left(q^{5}, q^{3}, q^{3}, q^{3} ; q^{4}\right)_{\infty}}{\left(q^{2}, q^{4}, q^{4}, q^{4} ; q^{4}\right)_{\infty}}
$$

Here and in what follows, $(a ; q)_{n}=(1-a)(1-a q) \cdots\left(1-a q^{n-1}\right)$ is the $q$-shifted factorial, and $[n]:=[n]_{q}=\left(1-q^{n}\right) /(1-q)$ denotes the $q$-integer. For simplicity, we also write $\left(a_{1}, a_{2}, \ldots, a_{m} ; q\right)_{n}=\left(a_{1} ; q\right)_{n}\left(a_{2} ; q\right)_{n} \cdots\left(a_{m} ; q\right)_{n}$ for $n \geqslant 0$ or $n=\infty$. 
For any odd prime $p$, let $\Gamma_{p}(x)$ be the $p$-adic Gamma function [17]. In 2015, Swisher [20] proved the following $p$-adic analogue of (1.1):

$$
\sum_{k=0}^{(p-1) / 4}(8 k+1) \frac{\left(\frac{1}{4}\right)_{k}^{4}}{k !^{4}} \equiv p \frac{\Gamma_{p}\left(\frac{1}{2}\right) \Gamma_{p}\left(\frac{1}{4}\right)}{\Gamma_{p}\left(\frac{3}{4}\right)} \quad\left(\bmod p^{3}\right), \quad \text { if } p \equiv 1 \quad(\bmod 4),
$$

which was originally conjectured by Van Hamme [21, (G.2)]. Liu and Wang [15] showed that (1.4) can also be deduced from the following $q$-supercongruence:

$$
\sum_{k=0}^{(n-1) / 4}[8 k+1] \frac{\left(q ; q^{4}\right)_{k}^{4}}{\left(q^{4} ; q^{4}\right)_{k}^{4}} q^{2 k} \equiv \frac{\left(q^{2} ; q^{4}\right)_{(n-1) / 4}}{\left(q^{4} ; q^{4}\right)_{(n-1) / 4}}[n] q^{(1-n) / 4} \quad\left(\bmod [n] \Phi_{n}(q)^{2}\right)
$$

(for a more general form, see [8, Theorem 4.3]). On the other hand, Guo and Schlosser [7, Theorem 2 with $d=4$ ] proved that,

$$
\sum_{k=0}^{n-1}[8 k+1] \frac{\left(q ; q^{4}\right)_{k}^{4}}{\left(q^{4} ; q^{4}\right)_{k}^{4}} q^{2 k} \equiv 0 \quad\left(\bmod \Phi_{n}(q)^{2}\right) \quad \text { for } n \equiv 3 \quad(\bmod 4) .
$$

Here, $\Phi_{n}(q)$ is the $n$-th cyclotomic polynomial in $q$, which may be given by

$$
\Phi_{n}(q)=\prod_{\substack{1 \leqslant k \leqslant n \\ \operatorname{gcd}(n, k)=1}}\left(q-\zeta^{k}\right)
$$

where $\zeta$ is an $n$-th primitive root of unity. Moreover, two rational functions $A(q)$ and $B(q)$ in $q$ are called congruent modulo a polynomial $P(q)$, denoted by $A(q) \equiv B(q)(\bmod P(q))$, if $P(q)$ divides the numerator of the reduced form of $A(q)-B(q)$ in the polynomial ring $\mathbb{Z}[q]$.

Recently, Liu and Wang [16] proved that, modulo $[n] \Phi_{n}(q)^{2}$,

$$
\sum_{k=0}^{M}[6 k+1] \frac{\left(q ; q^{4}\right)_{k}\left(q ; q^{2}\right)_{k}^{3}}{\left(q^{2} ; q^{2}\right)_{k}\left(q^{4} ; q^{4}\right)_{k}^{3}} q^{k^{2}+k} \equiv\left\{\begin{array}{lll}
\frac{\left(q^{2} ; q^{4}\right)_{(n-1) / 4}}{\left(q^{4} ; q^{4}\right)_{(n-1) / 4}}\left[n q^{(1-n) / 4},\right. & \text { if } n \equiv 1 & (\bmod 4) \\
0, & \text { if } n \equiv 3 & (\bmod 4)
\end{array}\right.
$$

where $M=(n-1) / 2$ or $n-1$. They also gave the following generalization of the second case of (1.6) modulo $\Phi_{n}(q)^{2}$, for any positive integer $d \geqslant 2$ and positive odd integer $n$ with $n \equiv d+1(\bmod 2 d)$,

$$
\sum_{k=0}^{(n-1) / d}[3 d k+1] \frac{\left(q, e, q^{1+d} / e ; q^{2 d}\right)_{k}\left(q, q, q^{d-1} ; q^{d}\right)_{k}}{\left(q^{d}, e, q^{1+d} / e ; q^{d}\right)_{k}\left(q^{2 d}, q^{2 d}, q^{d+2} ; q^{2 d}\right)_{k}} q^{d k} \equiv 0 \quad\left(\bmod \Phi_{n}(q)^{2}\right) .
$$

For some other recent work on $q$-supercongruences, see [3, 5, 6, 9 [14, 18, 19, 22, 24].

The first aim of this paper is to establish the a stronger version of (1.7) for even $d$ as follows. 
Theorem 1.1. Let $d \geqslant 2$ be an even integer and $e$ an indeterminate. Let $n \equiv d+1$ $(\bmod 2 d)$ be a positive integer. Then

$$
\sum_{k=0}^{(d n+n-1) /(2 d)}[3 d k+1] \frac{\left(q, e, q^{1+d} / e ; q^{2 d}\right)_{k}\left(q, q, q^{d-1} ; q^{d}\right)_{k}}{\left(q^{d}, e, q^{1+d} / e ; q^{d}\right)_{k}\left(q^{2 d}, q^{2 d}, q^{d+2} ; q^{2 d}\right)_{k}} q^{d k} \equiv 0 \quad\left(\bmod \Phi_{n}(q)^{3}\right)
$$

Note that the $q$-supercongruence (1.8) modulo $\Phi_{n}(q)^{2}$ also follows from (1.7), since the $k$-th summand on the left-hand side of (1.7) is congruent to 0 modulo $\Phi_{n}(q)^{2}$ for all $(n-1) / d<k \leqslant(d n+n-1) /(2 d)$. However, the $q$-supercongruence (1.7) does not hold modulo $\Phi_{n}(q)^{3}$ in general.

Letting $e \rightarrow 0$ and $e=-q$ in (1.8), respectively, we obtain

$$
\begin{gathered}
\sum_{k=0}^{(d n+n-1) /(2 d)}[3 d k+1] \frac{\left(q ; q^{2 d}\right)_{k}\left(q, q, q^{d-1} ; q^{d}\right)_{k}}{\left(q^{d} ; q^{d}\right)_{k}\left(q^{2 d}, q^{2 d}, q^{d+2} ; q^{2 d}\right)_{k}} q^{d\left(k^{2}+k\right) / 2} \equiv 0 \quad\left(\bmod \Phi_{n}(q)^{3}\right), \\
\sum_{k=0}^{(d n+n-1) /(2 d)}[3 d k+1] \frac{\left(q,-q,-q^{d} ; q^{2 d}\right)_{k}\left(q, q, q^{d-1} ; q^{d}\right)_{k}}{\left(q^{d},-q,-q^{d} ; q^{d}\right)_{k}\left(q^{2 d}, q^{2 d}, q^{d+2} ; q^{2 d}\right)_{k}} q^{d k} \equiv 0 \quad\left(\bmod \Phi_{n}(q)^{3}\right) .
\end{gathered}
$$

Letting $n=p$ be an odd prime and $q \rightarrow 1$ in each of the above $q$-supercongruences, we get the following result: for even $d \geqslant 2$ and $p \equiv d+1(\bmod 2 d)$,

$$
\sum_{k=0}^{(d p+p-1) /(2 d)}(3 d k+1) \frac{\left(\frac{1}{d}\right)_{k}^{2}\left(\frac{d-1}{d}\right)_{k}\left(\frac{1}{2 d}\right)_{k}}{k !^{3} 4^{k}\left(\frac{d+2}{2 d}\right)_{k}} \equiv 0 \quad\left(\bmod p^{3}\right) .
$$

For $d=4$, we have $\frac{d-1}{d}=\frac{d+2}{2 d}=\frac{3}{4}$. It is easy to see $\left(\frac{1}{2}\right)_{k} \equiv\left(\frac{1}{4}\right)_{k} \equiv 0(\bmod p)$ for $(3 p-1) / 4<k \leqslant p-1$. Thus, from the $d=4$ case of (1.9) we deduce the following supercongruence, which was conjectured by Liu and Wang [16, Conjecture 4].

Corollary 1.2. Let $p \equiv 5(\bmod 8)$ be a prime. Then

$$
\sum_{k=0}^{p-1}(12 k+1) \frac{\left(\frac{1}{4}\right)_{k}^{2}\left(\frac{1}{8}\right)_{k}}{k !^{3} 4^{k}} \equiv 0 \quad\left(\bmod p^{3}\right) .
$$

The second aim of this paper is to establish the following $q$-supercongruence, which is a generalization of [16, Theorm 8] for the second case with $d$ even.

Theorem 1.3. Let $d \geqslant 2$ be an even integer and $e$ an indeterminate. Let $n \equiv d+1$ $(\bmod 2 d)$ be a positive integer. Then, modulo $\Phi_{n}(q)^{3}$,

$$
\sum_{k=0}^{M}[3 d k-1] \frac{\left(q^{-1}, e, q^{d-1} / e ; q^{2 d}\right)_{k}\left(q, q, q^{d-3} ; q^{d}\right)_{k} q^{d k}}{\left(q^{d}, e, q^{d-1} / e ; q^{d}\right)_{k}\left(q^{2 d-2}, q^{2 d-2}, q^{d+2} ; q^{2 d}\right)_{k}} \equiv 0
$$

where $M=(n-1) / 2$ if $d=2$, and $M=(d n-n+1) /(2 d)$ otherwise. 
The third aim of this paper is to prove the following $q$-supercongruence, which was originally conjectured by Liu and Wang [16, Conjecture 5].

Theorem 1.4. Let $n$ be a positive odd integer. Then, modulo $\Phi_{n}(q)^{3}$,

$$
\begin{aligned}
& \sum_{k=0}^{(n-1) / 2}[6 k-1] \frac{\left(q^{-1} ; q^{4}\right)_{k}\left(q^{-1} ; q^{2}\right)_{k}\left(q ; q^{2}\right)_{k}^{2}}{\left(q^{2} ; q^{2}\right)_{k}\left(q^{4} ; q^{4}\right)_{k}\left(q^{2} ; q^{4}\right)_{k}^{2}} q^{k^{2}+k+1} \\
& \equiv\left\{\begin{array}{lll}
-\frac{\left(q^{2} ; q^{4}\right)_{(n-1) / 4}}{\left(q^{4} ; q^{4}\right)_{(n-1) / 4}} q^{(n-1) / 4}, & \text { if } n \equiv 1 & (\bmod 4), \\
0, & \text { if } n \equiv 3 & (\bmod 4) .
\end{array}\right.
\end{aligned}
$$

Liu and Wang originally conjectured that (1.11) holds modulo $[n] \Phi_{n}(q)^{2}$, which is not true (the first counterexample is $n=15$ ).

We shall prove Theorems 1.1, 1.3, and 1.4 by using the method of 'creative microscoping', which was introduced by the author and Zudilin [10]. At the end of this paper, we put forward several open problems on supercongruences and $q$-supercongruences.

\section{Proof of Theorem 1.1}

Recall that the basic hypergeometric series ${ }_{r+1} \phi_{r}$ (see Gasper and Rahman's monograph [4]) is defined as

$$
{ }_{r+1} \phi_{r}\left[\begin{array}{c}
a_{1}, a_{2}, \ldots, a_{r+1} \\
b_{1}, b_{2}, \ldots, b_{r}
\end{array} ; q, z\right]=\sum_{k=0}^{\infty} \frac{\left(a_{1}, a_{2}, \ldots, a_{r+1} ; q\right)_{k} z^{k}}{\left(q, b_{1}, \ldots, b_{r} ; q\right)_{k}} .
$$

We need a quadratic transformation of Rahman [4, (3.8.13)], which can be stated as follows:

$$
\begin{aligned}
& \sum_{k=0}^{\infty} \frac{\left(1-a q^{3 k}\right)\left(a, d, a q / d ; q^{2}\right)_{k}(b, c, a q / b c ; q)_{k}}{(1-a)(a q / d, d, q ; q)_{k}\left(a q^{2} / b, a q^{2} / c, b c q ; q^{2}\right)_{k}} q^{k} \\
& =\frac{\left(a q^{2}, b q, c q, a q^{2} / b c ; q^{2}\right)_{\infty}}{\left(q, a q^{2} / b, a q^{2} / c, b c q ; q^{2}\right)_{\infty}}{ }_{3} \phi_{2}\left[\begin{array}{c}
b, c, a q / b c \\
d q, a q^{2} / d
\end{array} ; q^{2}, q^{2}\right]
\end{aligned}
$$

provided that $d$ or $a q / d$ is not of the form $q^{-2 n}$ ( $n$ is a non-negative integer).

We first give a generalization of Theorem 1.1 with an extra parameter $a$. Note that this $q$-congruence modulo $\left(1-a q^{n}\right)\left(a-q^{n}\right)$ was already indicated by Liu and Wang [16]. In order to make the paper self-contained, we give a complete proof here.

Theorem 2.1. Let $d \geqslant 2$ be an even integer and a, e indeterminates. Let $n \equiv d+1$ $(\bmod 2 d)$ be a positive integer. Then, modulo $\Phi_{n}(q)\left(1-a q^{n}\right)\left(a-q^{n}\right)$,

$$
\sum_{k=0}^{(d n+n-1) /(2 d)}[3 d k+1] \frac{\left(q, e, q^{1+d} / e ; q^{2 d}\right)_{k}\left(a q, q / a, q^{d-1} ; q^{d}\right)_{k}}{\left(q^{d}, e, q^{1+d} / e ; q^{d}\right)_{k}\left(a q^{2 d}, q^{2 d} / a, q^{d+2} ; q^{2 d}\right)_{k}} q^{d k} \equiv 0 .
$$


Proof. Put $q \mapsto q^{d}, a=q^{1-d n-n}, b=a q, c=q / a$ and $d=e$ in Rahman's transformation (2.1). Then, for $n \equiv d+1(\bmod 2 d)$, we have

$$
\begin{aligned}
& \sum_{k=0}^{(d n+n-1) /(2 d)} \frac{\left(1-q^{3 d k+1-d n-n}\right)\left(q^{1-d n-n}, e, q^{1+d-d n-n} / e ; q^{2 d}\right)_{k}\left(a q, q / a, q^{d-1-d n-n} ; q^{d}\right)_{k}}{\left(1-q^{1-d n-n}\right)\left(q^{d}, e, q^{1+d-d n-n} / e ; q^{d}\right)_{k}\left(a q^{2 d-d n-n}, q^{2 d-d n-n} / a, q^{d+2} ; q^{2 d}\right)_{k}} q^{d k} \\
& =\frac{\left(q^{2 d+1-d n-n}, q^{2 d-1-d n-n}, a q^{d+1}, q^{d+1} / a ; q^{2 d}\right)_{\infty}}{\left(q^{d}, q^{d+2}, a q^{2 d-d n-n}, q^{2 d-d n-n} / a ; q^{2 d}\right)_{\infty}} \frac{\left(a q, q / a, q^{d-1-d n-n} ; q^{2 d}\right)_{k}}{\left(q^{2 d}, e q^{d}, q^{2 d+1-d n-n} / e ; q^{2 d}\right)_{k}} q^{2 d k} \\
& =0
\end{aligned}
$$

where we have used $\left(q^{2 d+1-d n-n} ; q^{2 d}\right)_{\infty}=0$ for $n \equiv d+1(\bmod 2 d)$. It is easy to see that, for $0 \leqslant k \leqslant(d n+n-1) /(2 d)$, the polynomial

$$
\left(1-q^{1-d n-n}\right)\left(q^{d}, e, q^{1+d-d n-n} / e ; q^{d}\right)_{k}\left(a q^{2 d-d n-n}, q^{2 d-d n-n} / a, q^{d+2} ; q^{2 d}\right)_{k}
$$

is relatively prime to $\Phi_{n}(q)$. Since $q^{n} \equiv 1\left(\bmod \Phi_{n}(q)\right)$, from (2.3) we deduce that the $q$-congruence (2.2) is true modulo $\Phi_{n}(q)$.

On the other hand, letting $q \mapsto q^{d}, a=q, b=q^{1-n}, c=q^{1+n}$ and $d=e$ in (2.1), we get

$$
\begin{aligned}
& \sum_{k=0}^{(n-1) / d}[3 d k+1] \frac{\left(q, e, q^{1+d} / e ; q^{2 d}\right)_{k}\left(q^{1-n}, q^{1+n}, q^{d-1} ; q^{d}\right)_{k}}{\left(q^{d}, e, q^{1+d} / e ; q^{d}\right)_{k}\left(q^{2 d-n}, q^{2 d+n}, q^{d+2} ; q^{2 d}\right)_{k}} q^{d k} \\
& =\frac{\left(q^{2 d+1}, q^{d+1-n}, q^{d+1+n}, q^{2 d-1} ; q^{2 d}\right)_{\infty}}{\left(q^{d}, q^{2 d+n}, q^{2 d-n}, q^{d+2} ; q^{2 d}\right)_{\infty}} \sum_{k=0}^{\infty} \frac{\left(q^{1-n}, q^{1+n}, q^{d-1} ; q^{2 d}\right)_{k}}{\left(q^{2 d}, e q^{d}, q^{2 d+1} / e ; q^{2 d}\right)_{k}} q^{2 d k} \\
& =0
\end{aligned}
$$

since $\left(q^{d+1-n} ; q^{2 d}\right)_{\infty}=0$ for $n \equiv d+1(\bmod 2 d)$. Noticing that $(d n-1) /(2 d)>(n-1) / d$, we conclude that the left-hand side of (2.2) is equal to 0 for $a=q^{-n}$ and $a=q^{n}$. Namely, the $q$-congruence (2.2) is true modulo $1-a q^{n}$ and $a-q^{n}$. Since $\Phi_{n}(q), 1-a q^{n}$, and $a-q^{n}$

are pairwise relatively prime polynomials in $q$, we complete the proof.

Proof of Theorem 1.1. Since $n \equiv d+1(\bmod 2 d)$, we have $\operatorname{gcd}(2 d, n)=1$. Hence, $\left(q^{2 d} ; q^{2 d}\right)_{k}$ is relatively prime to $\Phi_{n}(q)$ for any $0 \leqslant k \leqslant n-1$. It is clear that $(d n+$ $n-1) /(2 d) \leqslant n-1$. Moreover, the polynomial $1-q^{n}$ contains the factor $\Phi_{n}(q)$. The proof of (1.8) then follows from (2.2) by taking $a=1$.

\section{Proof of Theorem 1.3}

Like before, we first establish the following generalization of Theorem 1.3 with an additional parameter $a$. 
Theorem 3.1. Let $d \geqslant 2$ be an even integer and a,e indeterminates. Let $n \equiv d+1$ $(\bmod 2 d)$ be a positive integer. Then, modulo $\Phi_{n}(q)\left(1-a q^{n}\right)\left(a-q^{n}\right)$,

$$
\sum_{k=0}^{M}[3 d k-1] \frac{\left(q^{-1}, e, q^{d-1} / e ; q^{2 d}\right)_{k}\left(a q, q / a, q^{d-3} ; q^{d}\right)_{k} q^{d k}}{\left(q^{d}, e, q^{d-1} / e ; q^{d}\right)_{k}\left(a q^{2 d-2}, q^{2 d-2} / a, q^{d+2} ; q^{2 d}\right)_{k}} \equiv 0
$$

where $M=(n-1) / 2$ if $d=2$, and $M=(d n-n+1) /(2 d)$ otherwise.

Proof. Set $q \mapsto q^{d}, a=q^{-1-d n+n}, b=a q, c=q / a$ and $d=e$ in (2.1). Then, for $n \equiv d+1$ $(\bmod 2 d)$, we have

$$
\begin{aligned}
& \sum_{k=0}^{(d n-n+1) /(2 d)} \frac{\left(1-q^{3 d k-1-d n+n}\right)\left(q^{-1-d n+n}, e, q^{d-1-d n+n} / e ; q^{2 d}\right)_{k}\left(a q, q / a, q^{d-3-d n+n} ; q^{d}\right)_{k}}{\left(1-q^{-1-d n+n}\right)\left(q^{d}, e, q^{d-1-d n+n} / e ; q^{d}\right)_{k}\left(a q^{2 d-2-d n+n}, q^{2 d-2-d n+n} / a, q^{d+2} ; q^{2 d}\right)_{k}} q^{d k} \\
& =\frac{\left(q^{2 d-1-d n+n}, q^{2 d-3-d n+n}, a q^{d+1}, q^{d+1} / a ; q^{2 d}\right)_{\infty}}{\left(q^{d}, q^{d+2}, a q^{2 d-d n+n}, q^{2 d-d n+n} / a ; q^{2 d}\right)_{\infty}} \sum_{k=0}^{\infty} \frac{\left(a q, q / a, q^{d-3-d n+n} ; q^{2 d}\right)_{k}}{\left(q^{2 d}, e q^{d}, q^{2 d-1-d n+n} / e ; q^{2 d}\right)_{k}} q^{2 d k} \\
& =0
\end{aligned}
$$

where we have utilized $\left(q^{2 d-1-d n+n} ; q^{2 d}\right)_{\infty}=0$ for $n \equiv d+1(\bmod 2 d)$. Moreover, it is not difficult to see that $\left(q^{2 d-2} ; q^{2 d}\right)_{k}$ is relatively prime to $\Phi_{n}(q)$ for $0 \leqslant k \leqslant M$ (in fact, this is true for $0 \leqslant k \leqslant(d-1)(n-1) / d)$. Noticing $q^{n} \equiv 1\left(\bmod \Phi_{n}(q)\right)$ again, the modulus $\Phi_{n}(q)$ case of the $q$-congruence (3.1) follows from (3.2) immediately (for $d=2$, we need to use the fact that $\left(q^{-1} ; q^{4}\right)_{k} \equiv 0\left(\bmod \Phi_{n}(q)\right)$ for $\left.(n+1) / 4<k \leqslant(n-1) / 2\right)$.

On the other hand, letting $q \mapsto q^{d}, a=q^{-1}, b=q^{1-n}, c=q^{1+n}$ and $d=e$ in (2.1), we obtain

$$
\begin{aligned}
& \sum_{k=0}^{(n-1) / d}[3 d k-1] \frac{\left(q^{-1}, e, q^{d-1} / e ; q^{2 d}\right)_{k}\left(q^{1-n}, q^{1+n}, q^{d-3} ; q^{d}\right)_{k}}{\left(q^{d}, e, q^{d-1} / e ; q^{d}\right)_{k}\left(q^{2 d-2-n}, q^{2 d-2+n}, q^{d+2} ; q^{2 d}\right)_{k}} q^{d k} \\
& \quad=-\frac{\left(q^{2 d-1}, q^{2 d-3}, q^{d+1-n}, q^{d+1+n} ; q^{2 d}\right)_{\infty}}{q\left(q^{d}, q^{d+2}, q^{2 d-2-n}, q^{2 d-2+n} ; q^{2 d}\right)_{\infty}} \sum_{k=0}^{(n-1) /(2 d)} \frac{\left(q^{1-n}, q^{1+n}, q^{d-3} ; q^{2 d}\right)_{k}}{\left(q^{2 d}, e q^{d}, q^{2 d-1} / e ; q^{2 d}\right)_{k}} q^{2 d k} \\
& \quad=0
\end{aligned}
$$

as was first given by Liu and Wang [16]. In view of $(d n-n-1) /(2 d)<(n-1) / 2$ for $d=2$, and $(d n-n-1) /(2 d)>(n-1) / d$ for $d \geqslant 4$, one sees that the left-hand side of (3.1) is equal to 0 for $a=q^{-n}$ and $a=q^{n}$. Thus, the $q$-congruence (3.1) holds modulo $1-a q^{n}$ and $a-q^{n}$. Since the polynomials $\Phi_{n}(q), 1-a q^{n}$, and $a-q^{n}$ are relatively prime to one another, we accomplish the proof.

Proof of Theorem 1.3. In the proof of Theorem [3.1, we have mentioned that $\left(q^{2 d-2} ; q^{2 d}\right)_{k}$ is relatively prime to $\Phi_{n}(q)$ for $0 \leqslant k \leqslant(d n-n+1) /(2 d)$. The proof of (1.10) then follows from the $a=1$ case of (3.1). 


\section{Proof of Theorem 1.4}

We require the following result, which was first given in [5, Lemma 2.1]. For the reader's convenience, we include a short proof here.

Lemma 4.1. Let $n$ be a positive odd integer and a an indeterminate. Then

$$
\left(a q, q / a ; q^{2}\right)_{(n-1) / 2} \equiv(-1)^{(n-1) / 2} \frac{\left(1-a^{n}\right) q^{\left(1-n^{2}\right) / 4}}{(1-a) a^{(n-1) / 2}} \quad\left(\bmod \Phi_{n}(q)\right) .
$$

Proof. It is easy to see that

$$
\begin{aligned}
\left(q / a ; q^{2}\right)_{(n-1) / 2} & =(1-q / a)\left(1-q^{3} / a\right) \cdots\left(1-q^{n-2} / a\right) \\
& \equiv\left(1-q^{1-n} / a\right)\left(1-q^{3-n} / a\right) \cdots\left(1-q^{-2} / a\right) \\
& =(-1)^{(n-1) / 2}\left(a q^{2} ; q^{2}\right)_{(n-1) / 2} \frac{q^{\left(1-n^{2}\right) / 4}}{a^{(n-1) / 2}} \quad\left(\bmod \Phi_{n}(q)\right) .
\end{aligned}
$$

Hence, the left-hand side of (4.1) is congruent to

$$
(-1)^{(n-1) / 2}(a q ; q)_{n-1} \frac{q^{\left(1-n^{2}\right) / 4}}{a^{(n-1) / 2}} .
$$

For any $n$-th primitive root of unity $\zeta$, we have

$$
(a \zeta ; \zeta)_{n-1}=\frac{(a ; \zeta)_{n}}{1-a}=\frac{1-a^{n}}{1-a}
$$

and so $(a q ; q)_{n-1}$ is congruent to $\left(1-a^{n}\right) /(1-a)$ modulo $\Phi_{n}(q)$. This completes the proof.

We have the following parametric generalization of Theorem 1.4 for $n \equiv 1(\bmod 4)$.

Theorem 4.2. Let $n \equiv 1(\bmod 4)$ be a positive integer and a an indeterminate. Then, modulo $\Phi_{n}(q)\left(1-a q^{n}\right)\left(a-q^{n}\right)$,

$$
\sum_{k=0}^{(n-1) / 2}[6 k-1] \frac{\left(q^{-1} ; q^{4}\right)_{k}\left(q^{-1}, a q, q / a ; q^{2}\right)_{k}}{\left(q^{2} ; q^{2}\right)_{k}\left(q^{4}, a q^{2}, q^{2} / a ; q^{4}\right)_{k}} q^{k^{2}+k+1} \equiv \frac{\left(q^{2} ; q^{4}\right)_{(n-1) / 4}}{\left(q^{4} ; q^{4}\right)_{(n-1) / 4}} q^{(n-1) / 4} .
$$

Proof. Letting $d \rightarrow 0$ in (2.1), we have

$$
\sum_{k=0}^{\infty} \frac{\left(1-a q^{3 k}\right)\left(a ; q^{2}\right)_{k}(b, c, a q / b c ; q)_{k}}{(1-a)(q ; q)_{k}\left(a q^{2} / b, a q^{2} / c, b c q ; q^{2}\right)_{k}} q^{\left(k^{2}+k\right) / 2}=\frac{\left(a q^{2}, b q, c q, a q^{2} / b c ; q^{2}\right)_{\infty}}{\left(q, a q^{2} / b, a q^{2} / c, b c q ; q^{2}\right)_{\infty}}
$$


We then take $q \mapsto q^{2}, a=q^{-1-n}, b=a q, c=q / a$ in the above formula to obtain

$$
\begin{aligned}
& \sum_{k=0}^{(n+1) / 2} \frac{\left(1-q^{6 k-1-n}\right)\left(q^{-1-n} ; q^{4}\right)_{k}\left(a q, q / a, q^{-1-n} ; q^{2}\right)_{k}}{\left(1-q^{-1-n}\right)\left(q^{2} ; q^{2}\right)_{k}\left(a q^{2-n}, q^{2-n} / a, q^{4} ; q^{4}\right)_{k}} q^{k^{2}+k} \\
& =\frac{\left(q^{3-n}, a q^{3}, q^{3} / a, q^{1-n} ; q^{4}\right)_{\infty}}{\left(q^{2}, q^{2-n} / a, a q^{2-n}, q^{4} ; q^{4}\right)_{\infty}} \\
& =0 .
\end{aligned}
$$

Since $q^{n} \equiv 1\left(\bmod \Phi_{n}(q)\right)$, we conclude from the above equality that

$$
\sum_{k=0}^{(n+1) / 2}[6 k-1] \frac{\left(q^{-1} ; q^{4}\right)_{k}\left(q^{-1}, a q, q / a ; q^{2}\right)_{k}}{\left(q^{2} ; q^{2}\right)_{k}\left(q^{4}, a q^{2}, q^{2} / a ; q^{4}\right)_{k}} q^{k^{2}+k+1} \equiv 0 \quad\left(\bmod \Phi_{n}(q)\right)
$$

Namely,

$$
\begin{aligned}
& \sum_{k=0}^{(n-1) / 2}[6 k-1] \frac{\left(q^{-1} ; q^{4}\right)_{k}\left(q^{-1}, a q, q / a ; q^{2}\right)_{k}}{\left(q^{2} ; q^{2}\right)_{k}\left(q^{4}, a q^{2}, q^{2} / a ; q^{4}\right)_{k}} q^{k^{2}+k+1} \\
& \equiv-[2] \frac{\left(q^{-1} ; q^{4}\right)_{(n+1) / 2}\left(q^{-1}, a q, q / a ; q^{2}\right)_{(n+1) / 2}}{\left(q^{2} ; q^{2}\right)_{(n+1) / 2}\left(q^{4}, a q^{2}, q^{2} / a ; q^{4}\right)_{(n+1) / 2}} q^{(n+1)(n+3) / 4+1} \quad\left(\bmod \Phi_{n}(q)\right)
\end{aligned}
$$

By Lemma 4.1, we have

$$
\begin{aligned}
\frac{\left(a q, q / a ; q^{2}\right)_{(n+1) / 2}}{\left(a q^{2}, q^{2} / a ; q^{4}\right)_{(n+1) / 2}} & =\frac{\left(a q, q / a ; q^{2}\right)_{(n-1) / 2}\left(1-a q^{n}\right)\left(1-q^{n} / a\right)}{\left(a q^{2}, q^{2} / a ; q^{4}\right)_{(n-1) / 2}\left(1-a q^{2 n}\right)\left(1-q^{2 n} / a\right)} \\
& =q^{\left(n^{2}-1\right) / 4}\left(\bmod \Phi_{n}(q)\right) .
\end{aligned}
$$

Moreover, modulo $\Phi_{n}(q)$,

$$
\begin{aligned}
\frac{\left(q^{-1} ; q^{2}\right)_{(n+1) / 2}}{\left(q^{2} ; q^{2}\right)_{(n+1) / 2}} & =\frac{\left(1-q^{-1}\right)(1-q) \cdots\left(1-q^{n-2}\right)}{\left(1-q^{2}\right)\left(1-q^{4}\right) \cdots\left(1-q^{n+1}\right)} \equiv(-1)^{(n+1) / 2} q^{-(n+1)(n+3) / 4}, \\
-[2] \frac{\left(q^{-1} ; q^{4}\right)_{(n+1) / 2}}{\left(q^{4} ; q^{4}\right)_{(n+1) / 2}} q & \equiv \frac{\left(q^{3} ; q^{4}\right)_{(n-1) / 2}}{\left(q^{4} ; q^{4}\right)_{(n-1) / 2}}=\frac{\left(q^{3} ; q^{4}\right)_{(n-1) / 4}\left(q^{n+2} ; q^{4}\right)_{(n-1) / 4}}{\left(q^{4} ; q^{4}\right)_{(n-1) / 4}\left(q^{n+3} ; q^{4}\right)_{(n-1) / 4}} \\
& \equiv \frac{\left(q^{2} ; q^{4}\right)_{(n-1) / 4}}{\left(q^{4} ; q^{4}\right)_{(n-1) / 4}} .
\end{aligned}
$$

Employing the above three $q$-congruences, we see that the right-hand side of (4.5) reduces to

$$
\frac{\left(q^{2} ; q^{4}\right)_{(n-1) / 4}}{\left(q^{4} ; q^{4}\right)_{(n-1) / 4}} q^{(n-1) / 4} \quad\left(\bmod \Phi_{n}(q)\right) .
$$

This proves that (4.2) is true modulo $\Phi_{n}(q)$.

The modulus $\left(1-a q^{n}\right)\left(a-q^{n}\right)$ case of (4.2) was already given by Liu and Wang [16, (4.2) with $e \rightarrow 0$ ], and this can be easily checked by putting $q \mapsto q^{2}, a=q^{-1}, b=q^{1-n}, c=q^{1+n}$ in (4.3). Since the polynomials $\Phi_{n}(q)$ and $\left(1-a q^{n}\right)\left(a-q^{n}\right)$ are relatively prime, we finish the proof of the theorem. 
Proof of Theorem 1.4. Letting $a=1$ in (4.2), we arrive at (1.11) for $n \equiv 1(\bmod 4)$. On the other hand, letting $d=2$ and $e \rightarrow$ in (1.10), we are led to (1.11) for $n \equiv 3(\bmod 4)$.

\section{Concluding remarks and open problems}

Numerical calculation suggests that we can replace the upper bound of the sum in (1.9) by $p-1$. Namely, the following variation of (1.9) should be true.

Conjecture 5.1. Let $d \geqslant 2$ be an even integer and let $p \equiv d+1(\bmod 2 d)$ be a prime. Then

$$
\sum_{k=0}^{p-1}(3 d k+1) \frac{\left(\frac{1}{d}\right)_{k}^{2}\left(\frac{d-1}{d}\right)_{k}\left(\frac{1}{2 d}\right)_{k}}{k !^{3} 4^{k}\left(\frac{d+2}{2 d}\right)_{k}} \equiv 0 \quad\left(\bmod p^{3}\right)
$$

Furthermore, it seems that (1.8) is also true modulo $\Phi_{n}(q)^{3}$ for $N=n-1$, which we state as the following conjecture (which is also a generalization of Conjecture 5.1).

Conjecture 5.2. Let $d \geqslant 2$ be an even integer and $e$ an indeterminate. Let $n \equiv d+1$ $(\bmod 2 d)$ be a positive integer. Then

$$
\sum_{k=0}^{n-1}[3 d k+1] \frac{\left(q, e, q^{1+d} / e ; q^{2 d}\right)_{k}\left(q, q, q^{d-1} ; q^{d}\right)_{k}}{\left(q^{d}, e, q^{1+d} / e ; q^{d}\right)_{k}\left(q^{2 d}, q^{2 d}, q^{d+2} ; q^{2 d}\right)_{k}} q^{d k} \equiv 0 \quad\left(\bmod \Phi_{n}(q)^{3}\right)
$$

It should be pointed out that the $d=2$ case of (5.1) was already proved by Liu and Wang themselves [16, Theorem 1]. For $d=4$, since $\left(q^{d-1} ; q^{d}\right)_{k} /\left(q^{d+2} ; q^{2 d}\right)_{k}=1 /\left(-q^{3} ; q^{4}\right)_{k}$, one can easily see that each $k$-th summand on the left-hand side of (1.8) is congruent to 0 modulo $\Phi_{n}(q)^{3}$ for $(3 n-1) / 4<k \leqslant n-1$. Therefore, By Theorem 1.1, the $q$ supercongruence (5.1) is also true for $d=4$. However, the same arguments do not work for $d \geqslant 6$.

We find that Theorem 1.3 for $d=4$ can be further strengthened as follows.

Conjecture 5.3. Let $n \equiv 5(\bmod 8)$ be a positive integer and e an indeterminate. Then

$$
\sum_{k=0}^{(3 n+1) / 8}[12 k-1] \frac{\left(q^{-1}, e, q^{3} / e ; q^{8}\right)_{k}\left(q ; q^{4}\right)_{k}^{3} q^{d k}}{\left(q^{4}, e, q^{3} / e ; q^{4}\right)_{k}\left(q^{6} ; q^{8}\right)_{k}^{3}} \equiv 0 \quad\left(\bmod \Phi_{n}(q)^{4}\right)
$$

In particular, for any prime $p \equiv 5(\bmod 8)$,

$$
\sum_{k=0}^{(3 p+1) / 8}(12 k-1) \frac{\left(-\frac{1}{8}\right)_{k}\left(\frac{1}{4}\right)_{k}^{3}}{k ! 4^{k}\left(\frac{3}{4}\right)_{k}^{3}} \equiv 0 \quad\left(\bmod p^{4}\right)
$$


Recently, the author and Zudilin [11] have extended many classical $q$-supercongruences to the so-called Dwork-type $q$-supercongruences through a creative $q$-microscope. They also proposed several difficult conjectures on Dwork-type $q$-supercongruences. Here we would like to propose such extensions of (1.6) and (1.11) for $n \equiv 1(\bmod 4)$. We notice that a similar conjecture related to (1.5) was already made by Liu and Wang [15].

Conjecture 5.4. Let $n \equiv 1(\bmod 4)$ be a positive integer and let $r \geqslant 1$. Then, modulo $\left[n^{r}\right] \prod_{j=1}^{r} \Phi_{n^{j}}(q)^{2}$,

$$
\begin{aligned}
& \sum_{k=0}^{\left(n^{r}-1\right) / d}[6 k+1] \frac{\left(q ; q^{4}\right)_{k}\left(q ; q^{2}\right)_{k}^{3}}{\left(q^{2} ; q^{2}\right)_{k}\left(q^{4} ; q^{4}\right)_{k}^{3}} q^{k^{2}+k} \\
& \equiv \frac{\left(q^{2} ; q^{4}\right)_{\left(n^{r}-1\right) / 4}\left(q^{4 n} ; q^{4 n}\right)_{\left(n^{r-1}-1\right) / 4}}{\left(q^{4} ; q^{4}\right)_{\left(n^{r}-1\right) / 4}\left(q^{2 n} ; q^{4 n}\right)_{\left(n^{r-1}-1\right) / 4}}[n] q^{(1-n) / 4} \\
& \quad \times \sum_{k=0}^{\left(n^{r-1}-1\right) / d}[6 k+1] \frac{\left(q^{n} ; q^{4 n}\right)_{k}\left(q^{n} ; q^{2 n}\right)_{k}^{3}}{\left(q^{2 n} ; q^{2 n}\right)_{k}\left(q^{4 n} ; q^{4 n}\right)_{k}^{3}} q^{\left(k^{2}+k\right) n},
\end{aligned}
$$

where $d=1,2$.

Conjecture 5.5. Let $n \equiv 1(\bmod 4)$ be a positive integer and let $r \geqslant 1$. Then, modulo $\Phi_{n^{r}}(q) \prod_{j=1}^{r} \Phi_{n^{j}}(q)^{2}$,

$$
\begin{aligned}
& \sum_{k=0}^{\left(n^{r}-1\right) / 2}[6 k-1] \frac{\left(q^{-1} ; q^{4}\right)_{k}\left(q^{-1} ; q^{2}\right)_{k}\left(q ; q^{2}\right)_{k}^{2}}{\left(q^{2} ; q^{2}\right)_{k}\left(q^{4} ; q^{4}\right)_{k}\left(q^{2} ; q^{4}\right)_{k}^{2}} q^{k^{2}+k+1} \\
& \equiv \frac{\left(q^{2} ; q^{4}\right)_{\left(n^{r}-1\right) / 4}\left(q^{4 n} ; q^{4 n}\right)_{\left(n^{r-1}-1\right) / 4}}{\left(q^{4} ; q^{4}\right)_{\left(n^{r}-1\right) / 4}\left(q^{2 n} ; q^{4 n}\right)_{\left(n^{r-1}-1\right) / 4}} q^{(n-1) / 4} \\
& \quad \times \sum_{k=0}^{\left(n^{r-1}-1\right) / 2}[6 k-1] \frac{\left(q^{-n} ; q^{4 n}\right)_{k}\left(q^{-n} ; q^{2 n}\right)_{k}\left(q^{n} ; q^{2 n}\right)_{k}^{2}}{\left(q^{2 n} ; q^{2 n}\right)_{k}\left(q^{4 n} ; q^{4 n}\right)_{k}\left(q^{2 n} ; q^{4 n}\right)_{k}^{2}} q^{\left(k^{2}+k+1\right) n}
\end{aligned}
$$

\section{References}

[1] B.C. Berndt and R.A. Rankin, Ramanujan, Letters and Commentary, History of Mathematics 9, Amer. Math. Soc., Providence, RI; London Math. Soc., London, 1995.

[2] X. Chen and W. Chu, Hidden $q$-analogues of Ramanujan-like $\pi$-series, Ramanujan J. 54 (2021), 625-648.

[3] M. El Bachraoui, On supercongruences for truncated sums of squares of basic hypergeometric series, Ramanujan J. 54 (2021), 415-426.

[4] G. Gasper and M. Rahman, Basic Hypergeometric Series, second edition, Encyclopedia of Mathematics and Its Applications 96, Cambridge University Press, Cambridge, 2004.

[5] V.J.W. Guo, $q$-supercongruences modulo the fourth power of a cyclotomic polynomial via creative microscoping, Adv. Appl. Math. 120 (2020), Art. 102078. 
[6] V.J.W. Guo and J.-C. Liu, q-Analogues of two Ramanujan-type formulas for $1 / \pi$, J. Difference Equ. Appl. 24 (2018), 1368-1373.

[7] V.J.W. Guo and M.J. Schlosser, Some new q-congruences for truncated basic hypergeometric series: even powers, Results Math. 75 (2020), Art. 1.

[8] V.J.W. Guo and M.J. Schlosser, A new family of $q$-supercongruences modulo the fourth power of a cyclotomic polynomial, Results Math. 75 (2020), Art. 155.

[9] V.J.W. Guo and M.J. Schlosser, A family of $q$-supercongruences modulo the cube of a cyclotomic polynomial, Bull. Aust. Math. Soc., in press; https://doi.org/10.1017/S0004972721000630

[10] V.J.W. Guo and W. Zudilin, A q-microscope for supercongruences, Adv. Math. 346 (2019), 329-358.

[11] V.J.W. Guo and W. Zudilin, Dwork-type supercongruences through a creative $q$ microscope, J. Combin. Theory, Ser. A 178 (2021), Art. 105362.

[12] J-C. Liu, On a congruence involving $q$-Catalan numbers, C. R. Math. Acad. Sci. Paris 358 (2020), 211-215.

[13] J.-C. Liu and F. Petrov, Congruences on sums of $q$-binomial coefficients, Adv. Appl. Math. 116 (2020), Art. 102003.

[14] Y. Liu and X. Wang, q-Analogues of two Ramanujan-type supercongruences, J. Math. Anal. Appl. 502 (2021), Art. 125238.

[15] Y. Liu and X. Wang, q-Analogues of the (G.2) supercongruence of Van Hamme, Rocky Mountain J. Math. 51 (2021), 1329-1340.

[16] Y. Liu and X. Wang, Some $q$-supercongruences from a quadratic transformation by Rahman, Results Math. 77 (2022), Art. 44.

[17] Y. Morita, A p-adic supercongruence of the $\Gamma$ function, J. Fac. Sci. Univ. Tokyo 22 (1975), $255-266$.

[18] H.-X. Ni and L.-Y. Wang, Two $q$-supercongruences from Watson's transformation, Rev. R. Acad. Cienc. Exactas Fís. Nat. Ser. A Mat. 116 (2022), Art. 30.

[19] H. Song and C. Wang, Some $q$-supercongruences modulo the fifth power of a cyclotomic polynomial from squares of $q$-hypergeometric series, Results Math. 76 (2021), Art. 222.

[20] H. Swisher, On the supercongruence conjectures of van Hamme, Res. Math. Sci. 2 (2015), Art. 18.

[21] L. Van Hamme, Some conjectures concerning partial sums of generalized hypergeometric series, in: p-Adic Functional Analysis (Nijmegen, 1996), Lecture Notes in Pure and Appl. Math. 192, Dekker, New York, 1997, pp. 223-236.

[22] C. Wang, A new $q$-extension of the (H.2) congruence of Van Hamme for primes $p \equiv 1$ (mod 4), Results Math. 76 (2021), Art. 205.

[23] C. Wei, Some $q$-supercongruences modulo the fourth power of a cyclotomic polynomial, J. Combin. Theory, Ser. A 182 (2021), Art. 105469.

[24] W. Zudilin, Congruences for q-binomial coefficients, Ann. Combin. 23 (2019), 1123-1135. 